\title{
Drittes Kapitel Die Bildtheorie des Tractatus
}

Der zentrale Bestandteil der Wittgensteinschen Theorie des logischen Symbolismus ist die Bildtheorie: Durch den Begriff des Bildes, auf dem die Bildtheorie beruht, ist der Begriff des Symbols begründet. Das Bild, das ein Symbol ist, wird dadurch erfasst, dass man mit ihm operiert. Sofern die mit dem Bild ausführbaren Operationen als ihre Basen und Resultate Symbole haben, können sie als symbolische Operationen bezeichnet werden. Symbolische Operationen beschränken sich nicht auf Wahrheitsoperationen und Operationen des logischen Schließens, sondern umfassen weitere einige Komponenten solcher logischer Operationen bildende oder Voraussetzungen für ihre Anwendung schaffende Operationen, die Bestimmung deren Charakters zur Problematik gehört, die den Übergang Wittgensteins von der Theorie des Tractatus zu seiner Spätphilosophie bedingt. Dass ein logisches Bild (ein Satz) als Symbol charakterisiert werden kann, ist die Voraussetzung dafür, dass Prinzipien einer jeden im vorausgehenden Kapitel behandelten Theorie bestimmte Folgen auch für die Auffassung des logischen Symbolismus haben. Deswegen möchte ich in diesem Kapitel die Möglichkeit dieser Charakterisierung begründen und somit bestimmen, in welchem Sinn ein Satz ein Symbol ist.

\section{$\S 1$. Das Bild als Symbol}

Mit der Wahl des Terminus „Bild“, mit den Sätzen ,,in Bilderschrift“ über die Fechtenden in den Tagebüchern sowie mit den späteren Ausführungen über Unterschiede zwischen Portraits und Genrebildern gibt Wittgenstein Anlass dazu, dass viele Autoren die Analyse der Bildtheorie mit der Analyse des Begriffs des Bildes, als dessen Exemplifizierungen insbesondere gemalte Bilder betrachtet werden, verbinden.

Die meisten Autoren stimmen darin überein, dass logische Bilder Wittgensteins kaum mit sonstigen Bildern, insbesondere gemalten Bildern, vergleichbar sind, obwohl die vergleichende Analyse der beiden Bildbegriffe 
oft der Definition der Problematik, welche die Bildtheorie mit sich bringt, dient.

So sind laut Stenius (1960) logische Bilder von den sonstigen Bildern grundverschieden. Während das Bild Wittgensteins als Abbildung des Prototyps des Bildes nach einer bestimmten Zuordnungsvorschrift definierbar ist, so dass eine solche Abbildung im Fall der Wahrheit des Bildes eine isomorphe Abbildung ist ${ }^{1}$, beinhalten die sonstigen Bilder eher eine allgemeine Anweisung, wie eine Zuordnung möglich ist, als eine feste Vorschrift $^{2}$. Die aufgrund der Auffassung Stenius' definierbare Problematik der Bildtheorie besteht darin, dass die Interpretation des Satzes (des logischen Bildes), wenn sie an keine zur Kenntnis von Namen zusätzlichen Bedingungen gebunden ist, mehrere Zuordnungsvorschriften zulässt. Eine solche eine eindeutige Interpretation des Satzes festlegende Bedingung könnte nicht nur die Vorgabe einer bestimmten Zuordnungsvorschrift für alle in einer Sprache gebrauchten Namen sein, was voraussetzen würde, dass es sich bei der Sprache um eine formalisierte Sprache handelt, sondern auch in der Beschreibung der Gebrauchssituation des Satzes bestehen, wie es der Gebrauch des Begriffs des Sprachspiels voraussetzt.

Ähnliche Bedenken hat Fogelin (1976), der die Auffassung vertritt, dass die von den logischen Bildern verschiedenen Bilder sich den Forderungen einer logischen Theorie nicht richtig unterordnen lassen und insbesondere nicht richtig strukturiert sind, um die laut Fogelin Hauptidee der Bildtheorie vertreten zu können: Dass man eine Menge wechselseitig unabhängiger Sätze konstruieren kann, die wechselseitig unabhängige Sachlagen darstellen. Den Unterschied zwischen einem Bild im Sinne Wittgensteins und einem Bild, das sich nicht auf diese Weise charakterisieren lässt, sieht Fogelin vor allem darin, wie das jeweilige Bild sagt, dass etwas nicht der Fall ist. Während ein von Wittgensteins Definition abweichendes Bild das tun kann, indem es den Ausschluss einer bestimmten Beziehung zwischen gewissen Gegenständen dadurch exemplifiziert, dass es Dinge darstellt, die nicht in dieser Beziehung zu einander stehen, kann das Bild im Sinne Wittgensteins das nur dadurch tun, dass es gerade das darstellt, was ausge-

\footnotetext{
${ }^{1}$ E. Stenius. Wittgenstein's Tractatus. A Critical Exposition of its Main Lines of Thought (im weiteren: Stenius. Tractatus). Oxford, Basil Blackwell, 1964, 2.Aufl., 97 ${ }^{2}$ Ebd., 111-112
} 
schlossen wird und darüber hinaus sagt, dass es sich so nicht verhält ${ }^{3}$. Fogelin erkennt als eines der Probleme, welche die Bildtheorie birgt, den Umstand an, dass sich Wittgensteins Elementarsätze als abhängig voneinander erweisen können.

Dieses Problem erörtert Wittgenstein in bezug auf Farbaussagen ${ }^{4}$. Wittgenstein geht davon aus, dass Sätze wie „A ist rot" und „A ist grün“, in welchen „A“ dieselbe Bedeutung hat, nicht zugleich wahr sein können: Der Satz „A ist rot und A ist grün“ ist eine Kontradiktion oder sinnlos, obwohl nach Konstruktionsregeln dieser Satz die Wahrheitsgründe einer gewöhnlichen Konjunktion haben müsste, womit die Möglichkeit seiner Wahrheit zugelassen wäre. Das Problem dieser Sinnlosigkeit ist ein Problem zweier Theorien des Tractatus. Einerseits betrifft es die Theorie der Wahrheitsfunktionen, die auf der Idee der gegenseitigen Unabhängigkeit der Wahrheitswerte der Elementarsätze aufbaut. Andererseits betrifft dieses Problem die Bildtheorie: Die Konstruktion zusammengesetzter Sätze aus zwei Elementarsätzen ${ }^{5}$ erweist sich manchmal als abhängig von den Schlussfolge-

\footnotetext{
${ }^{3}$ R. J. Fogelin. Wittgenstein (im weiteren: Fogelin.Wittgenstein). London, Henley, Boston, Routledge \& Kegan Paul, 1976, 34-35

${ }^{4}$ Vgl. L. Wittgenstein. ,Some Remarks on Logical Form” (im weiteren: Some Remarks). In: I.M. Copi, R.W. Beard (Hrsg.). Essays on Wittgenstein's Tractatus. London, Routledge \& Kegan Paul, 1966, 31-38

${ }^{5}$ Dass der Satz „A ist rot“" als Beispiel eines Elementarsatzes betrachtet werden kann, wird aus unterschiedlichen Gründen bestritten. Einer der Gründe ist die Feststellung, dass Wittgenstein im Tractatus keine Beispiele von Gegenständen angibt. Ein anderer Grund ist der Satz 6.3751, der als Forderung nach einer weiteren Analyse von Farbaussagen gelesen wird. Wittgensteins Analyse der logischen Struktur der Farbaussagen im Aufsatz „Some Remarks on Logical Form“ (S. Some Remarks, 34-37) sowie in den Manuskripten aus den Jahren 1929-1932 zeigt, dass er solche Sätze als Beispiele von Elementarsätzen behandelt. Im Manuskript „VIII. Bemerkungen zu philosophischen Grammatik“ erkennt er ausdrücklich die Möglichkeit an, den Satz „Dieser Ort ist jetzt rot" als Elementarsatz aufzufassen, sofern dieser Satz keine Wahrheitsfunktion von anderen Sätzen ist oder als solche definiert wird. S. L. Wittgenstein. „VIII. Bemerkungen zur philosophischen Grammatik“ (im weiteren: VIII.Bemerkungen). In: L. Wittgenstein. Wiener Ausgabe 4, 103-240. Wien, New York, Springer, 1995, 237-238. Den Satz 6.3751 betrachte ich nicht als Forderung, den Satz „A ist rot“" weiter zu analysieren: Dass „die logische Struktur der Farbe“ die Möglichkeit ausschließt, ein und demselben Ort zugleich Rot und Grün zu prädizieren, gehört zum „Inhalt“ der Festlegungen, die bei der Konstruktion des logischen Symbolismus getroffen werden und die z.B. durch die Forderung ausgedrückt werden können, für verschiedene Gegenstände verschiedene Namen zu gebrauchen (s. unten, S.81). G.H. von Wright betrachtet die
} 
rungen, die es erlauben, von der Wahrheit eines der Elementarsätze auf die Falschheit des anderen zu schließen, was vor allem bedeutet, dass solche Elementarsätze keine eigentlichen Elementarsätze im Sinne des Tractatus sind. Die Quelle einer derartigen Sinnlosigkeit liegt nicht in der Struktur der zusammengesetzten Sätze, wie es bei Kontradiktionen der Fall ist, und kann auf zweifache Weise definiert werden. Einerseits, wenn man den Typenunterschied zwischen Namen anerkennt und auch Begriffswörter im Sinne Freges als Namen, nämlich Namen wahrheitswertiger Funktionen Begriffe, betrachtet, ist die gegenseitige Abhängigkeit mancher Elementarsätze und somit die Abhängigkeit der Konstruktion der Sätze von Schlussfolgerungen der Ausdruck der bestehenden logischen Beziehungen zwischen Begriffen, die einem durch Begriffswörter gegeben sind: Die Behauptung „A ist rot" bedeutet oder impliziert „A ist nicht grün“, weil Rot und Grün einander ausschließende Differenzierungen ein und desselben Begriffs und deswegen nicht logisch unabhängig voneinander sind. Ich bezeichne einen den Namen des Begriffs „,x ist rot“" enthaltenden Satz durch „ $\varphi_{1} x^{\prime \prime}$, den Satz der Gestalt ,x ist grün“ durch „, $\varphi_{2} x^{\prime \prime}$ und den Satz der Gestalt „,x ist farbig“ durch „ $\varphi x^{“}$ “. Wenn $\varphi x$ als Begriff definiert wird, der als ausschließende Disjunktion von endlich vielen (seien es $n$ Begriffe) , $\varphi_{\mathrm{i}} \mathrm{x}^{\text {“ }}$ darstellbar ist, wobei für $\mathrm{i}$ die Bedingung $1 \leq \mathrm{i} \leq \mathrm{n}$ erfüllt ist, lässt sich die Abhängigkeit zwischen den Begriffen dadurch charakterisieren, dass aus $\varphi_{1} \mathrm{x}$ die Implikation , $\varphi \mathrm{x} \supset \sim \varphi_{2} \mathrm{x}^{\prime}$ logisch folgt. Logisch unabhängig sind in einem solchen Fall in erster Linie Begriffe, welche die Rolle von Kategorien in dem Sinn erfüllen, dass keiner von ihnen in irgendwelchen derartige logische Schlüsse erlaubenden Beziehungen zu anderen Begriffen steht. Es besteht allerdings die Möglichkeit, den Begriff $\varphi x$ als eine nichtausschließende Disjunktion von $\mathrm{n}$ Begriffen zu definieren, wobei dann aus $\varphi_{1} \mathrm{x}$ logisch $\varphi \mathrm{x}$, aber nicht $\varphi_{2} \mathrm{x}$ oder $\sim \varphi_{2} \mathrm{x}$ folgt. Diese Möglichkeit ist ein Analogon der Möglichkeit, die von Wittgenstein in den Manuskripten aus den Jahren 1929-1932 analysiert wird und in seinem Vorschlag, jede Farbe

Tatsache, dass ein Gegenstand in einem Sachverhalt einer bestimmten Struktur vorkommen kann oder nicht kann (wobei man unter solche Tatsachen auch die Unmöglichkeit für Rot und Grün, an ein und demselben Ort zu ein und derselben Zeit zu sein, einordnen kann), als eine logische Möglichkeit, welche zu Tatsachen der Logik gehört. Von solchen Tatsachen handelt nach Meinung von Wrights die Modallogik des Tractatus. S. von Wright.Modal Logic, 196-197. 
als Kombination bestimmter Anteile von Grundfarben zu betrachten, zum Ausdruck kommt ${ }^{6}$. Andererseits, wenn man Begriffswörter als eine Art Kopulae auffasst und somit keine Typenunterschiede zwischen Namen anerkennt $^{7}$, betrifft die Problematik der gegenseitigen Abhängigkeit mancher

${ }^{6}$ S. L. Wittgenstein. „II. Philosophische Bemerkungen“ (im weiteren:

II.Bemerkungen). In: L. Wittgenstein. Wiener Ausgabe 1. Wien, New York, Springer, 1994, 37-196, 61. Wittgenstein analysiert diesen Vorschlag in einem weiteren Manuskript. S. VIII.Bemerkungen, 229-231.

${ }^{7}$ Th. Ricketts. „Pictures, Logic, and the Limits of Sense in Wittgenstein's Tractatus” (im weiteren: Ricketts.Pictures). In: H. Sluga, D.G. Stern (Hrsg.). The Cambridge Companion to Wittgenstein. Cambridge University Press, 1996, 59-99, 70, 72-73. Dass Wittgenstein "Qualitäten und Relationen" im Briefwechsel als Kopulae charakterisiert und z.B. eine Eigenschaft als „, $\exists \mathrm{x}) . \varphi \mathrm{x}$ “ darstellt und sie für etwas erklärt, das kein Komplex ist (s. Briefwechsel, 25-26), hat auch eine andere Bedeutung als diejenige, die mit dieser Auffassung Ricketts verbindet. In einem Brief, der im Sommer 1912 an Russell geschrieben wurde, identifiziert Wittgenstein die Kopula mit einem Schluss. In dem Ausdruck ,i[p, q, r] “ (,aus |- p und |- q folgt |- r“) ist i eine Kopula, die Komplexe mit einander verbindet. Den Ausdruck benutzt Wittgenstein zur Defini-

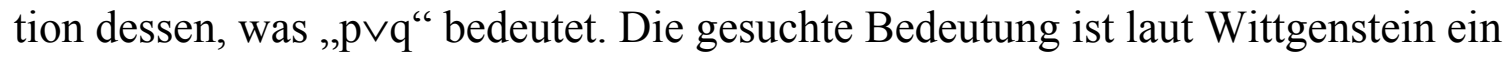
Komplex. Nach Wittgensteins Definition bedeutet , $\varepsilon_{1}(\mathrm{x}, \mathrm{y}) \vee \varepsilon_{2}(\mathrm{u}, \mathrm{z})$ “ Folgendes:

- $\left(\varepsilon_{1}(\mathrm{x}, \mathrm{y}), \varepsilon_{2}(\mathrm{u}, \mathrm{z}), \beta(\mathrm{x}, \mathrm{y}, \mathrm{u}, \mathrm{z})\right)$. i $\left[\varepsilon_{1}(\mathrm{x}, \mathrm{y}) ; \varepsilon_{2}(\mathrm{u}, \mathrm{z}) ; \beta(\mathrm{x}, \mathrm{y}, \mathrm{u}, \mathrm{z})\right]$

- $\left(\varepsilon_{1}(\mathrm{x}, \mathrm{y}), \varepsilon_{2}(\mathrm{u}, \mathrm{z}), \beta(\mathrm{x}, \mathrm{y}, \mathrm{u}, \mathrm{z})\right)$. i $\left[\sim \varepsilon_{1}(\mathrm{x}, \mathrm{y}) ; \varepsilon_{2}(\mathrm{u}, \mathrm{z}) ; \beta(\mathrm{x}, \mathrm{y}, \mathrm{u}, \mathrm{z})\right]$

- $\left(\varepsilon_{1}(\mathrm{x}, \mathrm{y}), \varepsilon_{2}(\mathrm{u}, \mathrm{z}), \beta(\mathrm{x}, \mathrm{y}, \mathrm{u}, \mathrm{z})\right)$. i $\left[\varepsilon_{1}(\mathrm{x}, \mathrm{y}) ; \sim \varepsilon_{2}(\mathrm{u}, \mathrm{z}) ; \beta(\mathrm{x}, \mathrm{y}, \mathrm{u}, \mathrm{z})\right]$

- $\left(\varepsilon_{1}(\mathrm{x}, \mathrm{y}), \varepsilon_{2}(\mathrm{u}, \mathrm{z}), \beta(\mathrm{x}, \mathrm{y}, \mathrm{u}, \mathrm{z})\right)$. i $\left[\sim \varepsilon_{1}(\mathrm{x}, \mathrm{y}) ; \sim \varepsilon_{2}(\mathrm{u}, \mathrm{z}) ; \beta(\mathrm{x}, \mathrm{y}, \mathrm{u}, \mathrm{z})\right]$

- $\beta(\mathrm{x}, \mathrm{y}, \mathrm{u}, \mathrm{z})$

Die Herausgeber des Briefwechsels McGuiness und von Wright gehen davon aus, dass dieser Ausdruck als Ausdruck der Disjunktion einen Fehler in der 4-ten Zeile enthält, der darin besteht, dass vor „ $\beta(\mathrm{x}, \mathrm{y}, \mathrm{u}, \mathrm{z})^{“}$ “ das Negationszeichen fehlt. S. Briefwechsel, 20. Der Fehler in der Darstellung ist dann enthalten, wenn der Ausdruck als Ausdruck der Wahrheitsbedingungen der Disjunktion gedeutet wird. Ich gehe jedoch davon aus, dass der Ausdruck die Bedingungen definiert, unter welchen $\beta(\mathrm{x}, \mathrm{y}, \mathrm{u}, \mathrm{z})$ als Disjunktion von $\varepsilon_{1}(\mathrm{x}, \mathrm{y})$ und $\varepsilon_{2}(\mathrm{u}, \mathrm{z})$ aus diesen beiden Sätzen logisch folgt. Der obige Ausdruck ist demnach eine Schlussfigur, deren letzter Satz der Schlusssatz ist. Als eine Tautologie geschrieben, hat diese Figur die Gestalt p.q. $\supset$.pvq. 1937 analysiert Lazerowitz die Anwendbarkeit der Matrix-Methode auf Tautologien und Kontradiktionen. Die Matrix-Methode ist für ihn die Methode des Aufzeigens der logischen Bedingungen, unter welchen eine gegebene Wahrheitsfunktion wahr sein kann. Weil laut Wittgenstein die Tautologie bedingungslos wahr ist, und in diesem Sinn keine Wahrheitsfunktion ist, ist auf sie die Matrix-Methode nicht anwendbar. Die Matrix, welche die Wahrheitsbedin-

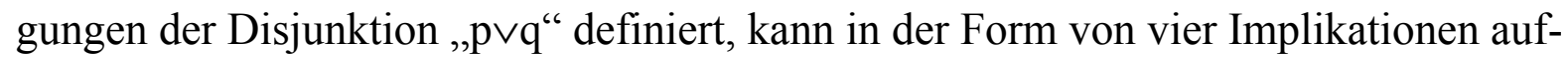
geschrieben werden:

$$
\text { p.q. } \supset . p \vee q
$$


Elementarsätze die Struktur und vor allem die Konstruktion der Sätze. Sowohl die Abgrenzung der Begriffe, die von der Bestimmung der Quelle der besagten Sinnlosigkeit als einer Begriffsbeziehung gefordert wird, als auch die Regelung der Struktur der Sätze verlangt Einschränkungen auf die An-

$$
\begin{aligned}
& \sim \text { p.q. } \supset . p \vee q \\
& \text { p. } \sim \text { q. } \supset \text {.p } \vee \mathrm{q} \\
& \sim \text { p. } \sim \text { q. } \supset \sim(p \vee q)
\end{aligned}
$$

Die Verneinung des Konsequens jeder der vier Implikationen führt zu einem Widerspruch zwischen dem Antezedens der Implikation und der Verneinung. Der Widerspruch kann durch die Eliminierung des Antezedens beseitigt werden. Die Wahrheitsbedingungen für eine Tautologie, die ich hinter der Wittgensteinschen Definition vermute, haben dagegen folgende Gestalt:

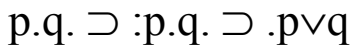

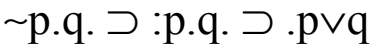

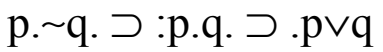

$$
\begin{aligned}
& \sim \text { p. q. } \supset \text { :p.q. } \supset \text {.p } \vee \mathrm{q}
\end{aligned}
$$

Einer der Thesen Lazerowitz' folgend, welche die Nicht-Anwendbarkeit der MatrixMethode auf Tautologien bestätigen soll, kann man behaupten: Bei der Verneinung des Konsequens jeder dieser Implikationen entsteht kein Widerspruch zwischen dem jeweiligen Antezedens und der Verneinung des tautologischen Satzes, der durch die Elimination des Antezedens der Implikation aufgehoben werden kann. S. M. Lazerowitz. "Tautologies and the Matrix Method". In: Mind 46, 1937, 191-205, 199-201. Der Grund: Der Satz ,p.q : p p. q“, der das Konsequens jeder der Implikationen verneint, ist ein Widerspruch unabhängig von seinen Wahrheitsbedingungen im Unterschied zu Verneinungen der Sätze ,p $\vee q “$ und , $\sim(p \vee q) “$. Meine Annahme über die Definition dessen, was ,p $\vee q^{\prime \prime}$ bedeutet, führt mich zu einer weiteren Hypothese. Wenn Qualitäten und Relationen Kopulae im Sinne einer Folgebeziehung sind, dann kann man z.B. Eigenschaften als Kopulae der Gestalt „i[p, q, r]“ definieren, so dass ein Satz ,$\varepsilon(\mathrm{x})$ “ Folgendes bedeutet:

$$
\begin{aligned}
& \text { |- }(\mathrm{y}, \varepsilon(\mathrm{x}), \beta(\mathrm{z})) \cdot \mathrm{i}[(\exists \mathrm{x}) \cdot \varepsilon(\mathrm{x}) ; \varepsilon(\mathrm{y}) \supset \beta(\mathrm{z}) ; \beta(\mathrm{z})] \\
& \mid-\beta(\mathrm{z})
\end{aligned}
$$

1912 geht Wittgenstein davon aus, dass das Zeichen eines verallgemeinerten Satzes kein vollständiges Symbol ist und nur in einem Schluss eine Bedeutung hat. S. Briefwechsel, 19. Durch den Ausdruck „, $\exists x) . \varphi x “$ stellt er eine Eigenschaft dar und erklärt den Ausdruck für nicht-komplex, womit ein Grund für die Anerkennung der logischen Formen im Sinne Russells als besonderer Objekte geschaffen wird. Wittgensteins Auffassung der Kopulae könnte trotz des späteren Aufgebens der These über einen nichtkomplexen Charakter des Ausdrucks der Form , $(\exists \mathrm{x}) . \varphi \mathrm{x}$ “, das mit der Anerkennung eines solchen Ausdrucks als eines sinnvollen Satzes einhergeht, sowohl die Behauptung des Tractatus, dass in einem Elementarsatz alle logischen Operationen enthalten sind (TLP, 5.47), als auch die Forderung, zwischen Satz und Namen zu unterscheiden, sofern der Satz auch dann nicht einfach sein kann, wenn er keine Namen enthält (Notes on Logic, 97), erklären. 
wendung der Ersetzungsregeln bei der Konstruktion des logischen Symbolismus: Man muss einige Ersetzungen von Namen in Elementarsätzen sowie gleichzeitige Einsetzungen ein und desselben Namens in die von einander abhängige Elementarsätze ergebenden Satzformen verbieten. Wenn man von dem allgemeinen Hinweis darauf, dass die Beziehungen zwischen Namen durch syntaktische Regeln des Symbolismus beschrieben werden, absieht, kann man Wittgensteins Forderung, für verschiedene Gegenstände verschiedene Zeichen zu gebrauchen ${ }^{8}$, als einen Versuch deuten, solche Einschränkungen explizit einzuführen. Selbst wenn diese Forderung als die Forderung aufgefasst wird, dass verschiedene Namen durch verschiedene Variablen und verschiedene Variablen durch verschiedene Namen ersetzt werden müssen, ist sie nicht hinreichend, um die beiden Sätze, "A ist rot" und "A ist grün", als Elementarsätze im Symbolismus beizubehalten, was man braucht, um verschiedene Weltbeschreibungen konstruieren zu können. Mit den durch diese Forderung eingeführten Einschränkungen kann man zwar das Ziehen des Schlusses ,Ein x erfüllt die Bedingung, dass x rot und $\mathrm{x}$ grün ist" aus dem Satz „A ist rot und B ist grün“ verhindern, aber nicht das gleichzeitige Einsetzen von „A“ in zwei Satzformen „, $\mathrm{x}$ ist rot“ und „, ist grün“. Eine der möglichen Lösungen dieser Problematik besteht in dem Aufgeben des Begriffs des Elementarsatzes ${ }^{9}$. Wie die Einführung des Begriffs der Familienähnlichkeiten zeigt, verzichtet Wittgenstein auch auf die Forderung nach der strengen Begriffsabgrenzung ${ }^{10}$ und sucht nach anderen Kriterien der Eindeutigkeit des Sinnes eines Satzes als die Erklärung der Bedeutung seiner Bestandteile.

Sofern der Vergleich der logischen Bilder mit den sonstigen Bildern eine Erklärung der Veränderung der Ansichten Wittgensteins ermöglicht, kann man diese vergleichende Betrachtungsweise der Bildbegriffe als eine fruchtbare Methode der Analyse ansehen. Der Grund für die Anwendbarkeit dieser Methode ist das Gemeinsame der beiden Bildbegriffe, das in dem Charakter der semiotischen Funktionen eines Bildes liegt.

\footnotetext{
${ }^{8}$ TLP, 3.33, 5.53-5.5321

${ }^{9}$ II.Bemerkungen, 50

${ }^{10}$ Zum Zusammenhang zwischen dem Begriff der Familienähnlichkeiten und dem Aufgeben der besagten Forderung s. z.B. Z.A. Sokuler. Das Problem der Begründung des Wissens (Gnoseologische Konzepte L. Wittgensteins und K. Poppers) (russ.) (im weiteren: Sokuler). Moskau, Nauka, 1988, 80.
} 
Eine der philosophischen Theorien, welche die vergleichende Betrachtungsweise der Bildbegriffe erlaubt, ist die Theorie Gadamers (1960) ${ }^{11}$, der davon ausgeht, dass die ästhetische Erfahrung kein Subsumieren des Angeschauten unter das Allgemeine ist, und deswegen den Begriff der Kunsterfahrung auf den Begriffen des Spiels und der Darstellung aufbaut.

Laut dieser Theorie ist das Kunstwerk ein Produkt schöpferischer Tätigkeit, das hergestellt wird und kraft seiner materiellen Verkörperung von seinem Verfasser abgelöst werden kann. Zugleich ist es kein Produkt, das als etwas Fertig-Seiendes nach Belieben genommen und weggelegt werden kann. Das Kunstwerk ist nach Gadamer ein Gebilde: das, was eine materielle Verkörperung hat und trotzdem von seinem Empfänger in der Kunsterfahrung konstruiert wird und in diesem Sinn in weiterer Bildung begriffen ist. Wie auch das Interesse an Kunst hat das Gebilde einen Spielcharakter. Bei der Bestimmung dieses Charakters geht Gadamer von der Theorie Huizingas aus, der das Spielen als ein freies, durch einen besonderen Spielraum abgegrenztes Handeln des Menschen nach festgelegten Regeln, dessen Zweck im Spiel selbst liegt, definiert ${ }^{12}$. Nach Gadamer ist das Spiel einer Verwandlung ins Gebilde fähig. Die Verwandlung ins Gebilde vollzieht sich beim Spielen des Spiels, wobei das Spiel zu einer Selbstdarstellung wird, in welcher das Spiel das ist, was gemeint und zugleich verstanden wird. Die Selbstdarstellung charakterisiert die Seinsweise des Spiels und ist deswegen das ontologische Moment seines Daseins. Eines der Merkmale der Verwandlung des Spiels in eine Selbstdarstellung ist es, dass der Spieler seine Identität verliert und zu einem Element des Spiels wird.

${ }^{11}$ H.-G. Gadamer. Wahrheit und Methode. Grundzüge einer philosophischen Hermeneutik (im weiteren: Wahrheit und Methode). In: H.-G. Gadamer. Gesammelte Werke 1. Tübingen, J.C.B. Mohr, 1986, s. auch die Aufsätze „Die Aktualität des Schönen. Kunst als Spiel, Symbol und Fest“ (im weiteren: Die Aktualität des Schönen). In: H.G. Gadamer. Gesammelte Werke 8. Tübingen, J.C.B. Mohr, 1993, 94-142, „Das Spiel der Kunst" (im weiteren: Das Spiel der Kunst). In: H.-G. Gadamer. Gesammelte Werke 8. Tübingen, J.C.B. Mohr, 1993, 86-93, „Text und Interpretation“. In: H.-G. Gadamer. Gesammelte Werke 2. Tübingen, J.C.B. Mohr, 1986, 330-360.

${ }^{12}$ J. Huizinga. Homo Ludens. Vom Ursprung der Kultur im Spiel (im weiteren: Homo Ludens). Reinbek bei Hamburg, Rowohlt Taschenbuch Verlag, 1987, 15-20 
Deswegen kann sich der Spieler nicht zum Spiel wie zu einem Gegenstand verhalten, so dass im Spiel der Vergleich mit der Wirklichkeit nicht zugelassen wird.

Sofern der Spielcharakter des Gebildes demnach darin besteht, dass sein Dasein eine Selbstdarstellung ist, ist das Kunstwerk als Gebilde ein Bild, das eine darstellende und selbstdarstellende Funktion erfüllt. Das Bild wird von Gadamer als eine besondere Form der Darstellung von Darstellungsformen Zeichen und Symbol unterschieden. Als Gegensatz zu einer Darstellung betrachtet Gadamer auch Abbild. Sofern nach Gadamer das in einem Bild Abgebildete mit dem in ihm Dargestellten nicht zusammenfällt, ist man geneigt, die darstellende und die abbildende Funktion des Bildes als seine entgegengesetzten semiotischen Funktionen zu betrachten. Dagegen spricht, dass Gadamer das Urbild als das im Bild Dargestellte charakterisiert $^{13}$. Eine mögliche Lösung dieses terminologischen Problems besteht darin, dass man erstens zwischen verschiedenen semiotischen Funktionen des Bildes unterscheidet, die Gadamer als Formen der Darstellung bezeichnet, und zweitens als Darstellung im eigentlichen Sinn eine besondere semiotische Funktion des Gebildes (seine Bild-Funktion) charakterisiert, die das Kunstwerk als Gebilde auszeichnet und es von einem Abbild, Zeichen und Symbol unterscheidet. Diese Lösung kann man dadurch begründen, dass Gadamer das Gebilde (das Kunstwerk) einem Text ähnlich behandelt, der verstanden werden will. Die Möglichkeit dieser Behandlung ist mit der Betrachtung des Kunstwerks als Produktes einer schöpferischen Tätigkeit gegeben. Diese Tätigkeit kann ihrerseits als eine sich im Kunstwerk und seiner Struktur vergegenständlichende Einheit verschiedener Arten der menschlichen Tätigkeit aufgefasst werden. Eine solche Auffassung vertritt z.B. Kagan (1975) ${ }^{14}$, der folgende Arten der menschlichen Tätigkeit als Komponenten der schöpferischen Tätigkeit unterscheidet: eine umgestaltende Tätigkeit, die den Zweck einer reellen oder imaginären Umgestaltung ihres Objekts verfolgt, eine erkennende Tätigkeit, die auf das Objekt mit dem Ziel des Gewinnens des Wissens von der Welt gerichtet ist, eine wertorientierte Tätigkeit, welche die Bestimmung der Bedeutung des Ob-

\footnotetext{
${ }^{13}$ Wahrheit und Methode, 417

${ }^{14}$ M.S. Kagan, „Künstlerische Tätigkeit als Informationssystem“ (russ.). In: M.S. Kagan. Das Verfahren einer Systemuntersuchung und geisteswissenschaftliches Wissen. Ausgewählte Aufsätze. Leningrad, Universität Leningrad Verlag, 1991, 208-239
} 
jekts für den Menschen anstrebt, und eine kommunikative Tätigkeit, die sich auf andere Menschen mit dem Ziel der Organisation ihrer gemeinsamen Tätigkeit richtet. Die Struktur des Kunstwerkes umfasst verschiedene Ebenen, die diesen Tätigkeitsarten entsprechen: gnoseologische Ebene, die sich als Thema des Werkes realisiert, axiologische Ebene, die sich als Idee des Werkes verwirklicht und zusammen mit seinem Thema seinen poetischen Inhalt bildet, die Ebene der Bildform, die der modellierenden oder umgestaltenden Tätigkeit entspricht und sich z.B. im Sujet des Werkes äußert, sowie eine zeichen-kommunikative Ebene - die äußere materielle Form des Werkes, die es zu einem Text oder Zeichen-System macht. Das Vorhandensein der zeichen-kommunikativen Ebene erlaubt es, jedes Gebilde in seiner semiotischen Funktion zu betrachten.

Nach Gadamer sind Extreme der semiotischen Funktionen des Bildes Zeichen und Symbol, die jeweils die Funktion einer reinen Verweisung und einer reinen Vertretung erfüllen. Das Gebilde als Bild (als Darstellung) erfüllt sowohl diese beiden Funktionen als auch die semiotische Funktion, die zwischen diesen beiden Extremen anzusiedeln ist. Die Funktion einer Verweisung erfüllt das Bild durch sein Abbild-Sein: Das Bild bezieht sich auf sein nicht gegenwärtiges, aber gemeintes Urbild, indem es das Urbild wiedergibt und somit auf dieses verweist. Deswegen kann man ein Abbild in Hinsicht auf seine semiotische Funktion als eine Differenzierung des Zeichens betrachten. Dank seiner abbildenden Funktion kann das Bild mit seinem Urbild auf ihre Ähnlichkeit hin verglichen werden. Das Urbild hat ein von der Existenz des Bildes unabhängiges Sein, selbst wenn das Bild auf sein Urbild so auswirkt, dass erst das Bild es zu einem Urbild macht.

Das Bild als Darstellung ist zwar auf sein Urbild bezogen und sagt etwas über dieses, aber das Dargestellte ist nicht mit dem Abgebildeten identisch, sofern das Dargestellte vom Bild nicht ontologisch ablösbar ist. Das Dargestellte wird in der Darstellung bildhaft: Dargestellt wird das, was im Gebilde selbst zu finden ist. Nach Gadamer ist das Dargestellte das Gebilde selbst in seiner Totalität: Die Kunsterfahrung ist eine Erkenntnis, die im Erfassen des Bekannten (des Gebildes) in seinem Wesen oder als etwas besteht ${ }^{15}$. Indem das Bild das von ihm Dargestellte als mit ihm gegenwärtig erscheinen lässt, erfüllt das Bild eine symbolische Funktion, denn das

${ }^{15}$ Die Aktualität des Schönen, 123, 128, Wahrheit und Methode, 117-119 
Symbol bewirkt dasselbe für das von ihm Symbolisierte. Im Unterschied zum Symbol, dessen ein jedes Unendliches und Unsinnliches als seiner semiotischen Form bedarf, verweist das Bild auf das Abgebildete, indem es etwas darüber sagt. Das Bild als Abbild verweist, aber im Vergleich zum Abbild stellt es sich selbst dar. Deswegen ist das Bild kein reines Zeichen (kein bloßes Abbild) und kein reines Symbol. Ein weiterer Unterschied des Gebildes vom Zeichen und Symbol besteht darin, dass das Gebilde seine Bedeutung nicht durch Stiftung erhält, die den Zeichen und Symbolen ihre Bedeutung verleiht, wenn sie diese als solche bekannt macht. Seine eigentliche selbstdarstellende Funktion erhält das Gebilde durch die Kunsterfahrung, die ihrerseits dem Spielen des Spiels analog ist.

Obwohl für Wittgenstein Namen und einige Symbole wie die allgemeine Form des Satzes an eine bestimmte Bedeutung und einen bestimmten Zweck gebunden sind und der Satz von der Situation seiner Formulierung ${ }^{16}$ und seines Gebrauchs unabhängig ist, kann man dem Satz dennoch in Abhängigkeit von dem konkreten Bezug des Satzes (auf eine Tatsache, seine eigenen Wahrheitsbedingungen und letztendlich andere Sätze) verschiedene semiotische Funktionen zusprechen. Obwohl der Satz, aus der Situation seines Gebrauchs gelöst, kein Gebilde im Sinne Gadamers ist, berücksichtigt doch die Auffassung Wittgensteins und insbesondere die weitere Entwicklung seiner Ansichten die Universalität und die Produktivität des Symbolsystems der Sprache, die mit „einem beschränkten Schatz von Konventionen und dementsprechend von Sprachgebilden unbeschränkt Mannigfaltiges" darstellen kann $^{17}$ und in diesem Sinn den Charakter eines Gebildes hat, das bei jedem Selbstdarstellen, das für die Sprache das Sprechen ist, einen neuen Sinn erhalten kann. Wenn man von dieser sich bereits im Tractatus andeutenden späteren Entwicklung der Ansichten Wittgensteins absieht, ist der Satz deswegen mit einem Gebilde im Sinne Gadamers vergleichbar, weil er in eine Beziehung zu anderen Sätzen gebracht

\footnotetext{
${ }^{16}$ Wie Wittgensteins Manuskripte zeigen, sieht er die Bedingung dafür, dass das Symbol verstanden wird, in der Kenntnis des Symbols. Vgl. L. Wittgenstein. ,VI. Philosophische Bemerkungen“ (im weiteren: VI.Bemerkungen). In: L. Wittgenstein. Wiener Ausgabe 3, Wien, New York, Springer, 1995, 147-334, 188. Diese Kenntnis kann man als Ergebnis der Stiftung des Symbols auffassen.

${ }^{17}$ K. Bühler. Sprachtheorie. Die Darstellungsfunktion der Sprache (im weiteren: Bühler.Sprachtheorie). Stuttgart, Gustav Fischer Verlag, 2. Aufl., 1965, 76, vgl. TLP, 4.027, 4.03.
} 
werden kann, z.B. dadurch, dass man aus ihm andere Sätze folgert oder konstruiert. Durch solche Beziehungen zu anderen Sätzen wird jeder Satz zum Ausgangspunkt für eine neue Sinn-Konstruktion (Konstruktion einer neuen Beschreibung der Welt). Das ermöglicht die Anwendung einiger Thesen Gadamers, insbesondere der These über die Unterscheidung der darstellenden Funktion eines Bildes von seiner abbildenden Funktion sowie der These über den unvollendeten Charakter eines Gebildes, auf die Rekonstruktion der Bildtheorie Wittgensteins. Sofern die Möglichkeit einer durch einen Satz bestimmten Sinn-Konstruktion nicht von demjenigen, der mit dem Satz operiert, sondern vom Satz selbst getragen wird, kann man den Satz (das logische Bild) auch als ein Symbol betrachten.

In Hinblick auf die Gadamersche Charakterisierung des Bildes sowie auf die oben formulierten Prinzipien der Bildtheorie kann man den Satz als Bild folgendermaßen charakterisieren:

1. Der Satz ist ein Abbild in bezug auf eine Tatsache: Er bildet die Tatsache $a b$, indem er von einem bestimmten Gegenstand (einem Element im Verband eines möglichen Sachverhaltes) sagt, wie dieser Gegenstand ist ${ }^{18}$. Als Abbild weist der Satz die Identität mit der Tatsache auf - die Identität der logischen Form, welche die Form der Abbildung ist. Diese Identität zeigt sich in erster Linie in der gleichen Multiplizität der Elemente des Satzes einerseits und der Elemente der Struktur der Tatsache (der Gegenstände in einem möglichen Sachverhalt) andererseits. Diese Multiplizität ist für die beiden Möglichkeiten, nämlich für das Bestehen des Sachverhaltes und für sein Nichtbestehen, sowie für den Sachverhalt selbst, falls er besteht, dieselbe.

2. Der Satz, der ein Abbild in bezug auf eine Tatsache ist, ist eine Darstellung in bezug auf seinen Sinn: Er teilt seinen Sinn mit oder stellt ihn dar. Die Voraussetzung dafür ist es, dass der Satz ein Abbild ist, denn das Abbild-Sein eines Satzes bestimmt seine Wahrheitsargumente. Als Darstellung weist der Satz einen Unterschied von dem von ihm Abgebildeten auf: Dieser Unterschied ist durch den logischen Charakter der abbildenden Beziehung bestimmt und besteht in

${ }^{18}$ TLP, 3.221 
der Bipolarität des Satzes (darin, dass der Sinn des Satzes mit der Tatsache übereinstimmt oder nicht).

3. Dass der Satz, der ein Bild ist, außerdem einen Ort im logischen Raum bestimmt, charakterisiert den Satz als Symbol. Ein Symbol zu sein oder zeigen zu können ist eine besondere semiotische Funktion des Satzes. Diese zeigende Funktion ist nach Wittgenstein dadurch bestimmt, dass der Satz Sinn hat. Sie ist dadurch dennoch nicht erschöpft, weil auch sinnlose Sätze wie Tautologien und Kontradiktionen sie erfüllen. Sie ist aber selbst für solche Sätze dadurch bestimmt, dass ihre Teilsätze Sinn haben: Hätten die Teilsätze keinen Sinn (keine Wahrheitsbedingungen), könnten auch Tautologien und Kontradiktionen keine Sätze und insbesondere keine logischen Sätze sein, die gerade als Grenzfall der Zeichenverbindung und deswegen als extremer Fall der Verbindung der Wahrheitsbedingungen definiert sind ${ }^{19}$. Dass der Satz die zeigende Funktion erfüllen kann, setzt voraus, dass der Satz ein Objekt ist, auf welchem bestimmte Operationen ausgeführt werden können und welches selbst Operationen mit Zeichen vorschreibt.

\section{$\S 1$ b. Das Bild als Symbol}

Die von Gadamer in seiner semiotischen Analyse des Gebildes verwendete Idee einer unendlichen vom Symbol vertretenen Wirklichkeit lässt sich bis zu einigen Thesen Cassirers zurückverfolgen. Cassirer, der unter einem Symbol jedes sinnliche Dasein und So-sein versteht, das Sinn verkörpert ${ }^{20}$, sieht die Aufgabe eines jeden Symbols im Erkennen, welches im Aufbau der Welt in ihrer Ordnung und ihrem So-sein besteht ${ }^{21}$. Die Funktion eines Symbols besteht darin, dass es nicht einfach die vorhandene Wirklichkeit abbildet, sondern sich an der Konstituierung des Wirklichen als des Einen und des Vielen beteiligt, wobei Eines des Vielen die Einheit der Bedeutung

\footnotetext{
${ }^{19}$ Ebd., 4.46, 4.466

${ }^{20}$ E. Cassirer. Philosophie der symbolischen Formen III. Darmstadt, Wissenschaftliche Buchgesellschaft, 1972, 5. Aufl., 109

${ }^{21}$ E. Cassirer. „Zur Logik des Symbolbegriffs“. In: E. Cassirer. Wesen und Wirkung des Symbolbegriffs. Darmstadt, Wissenschaftliche Buchgesellschaft, 1969, 4. Aufl., 201-230, 208-209
} 
ist. Die Rolle symbolischer Formen wie Begriffe und wissenschaftlicher Symbole besteht nach Cassirer darin, dass sie ein Prinzip (eine Regel) beinhalten, das nicht durch die Betrachtung eines begrenzten angeschauten Umfangs erklärbar ist, sondern die Grenzen des Gegebenen überschreitet. Ein Begriff oder ein wissenschaftliches Symbol steht somit für ein Prinzip, das ein allgemein, d.h. unbeschränkt anwendbares Verfahren, z.B. das Konstruktionsverfahren für eine Zahl, charakterisiert ${ }^{22}$.

Einer derjenigen, die unter dem Einfluss Cassirers arbeiten, ist Losev, der eine Theorie des Symbols als einer besonderen Kategorie des Zeichens entwickelt $(1976)^{23}$. Jedes Symbol ist nach Losev ein Zeichen, aber nicht jedes Zeichen ist ein Symbol. Ein Zeichen ist ein Symbol, nur wenn es unendlich viele Bedeutungen besitzt. Ein Symbol kann unendlich viele Individuen bezeichnen und deswegen ein Unendliches vertreten, weil es den Charakter und die Allgemeinheit eines Gesetzes hat, welches vorgibt, wie man Individuen konstruieren kann. Die Funktion des Symbols im Erkennen besteht darin, dass es als Funktion der Wirklichkeit auftritt in dem Sinn, in welchem auch eine mathematische Funktion als Funktion ihrer Argumente bezeichnet wird, und dass seine Anwendung auf die Konstruktion oder Umgestaltung der Wirklichkeit gerichtet ist. Das mathematische Symbol einer Reihe gibt z.B. das Gesetz der Konstruktion der Reihe vor und vertritt die Reihe, indem es bestimmt, wie jedes einzelne Element der Reihe zu konstruieren ist. Die Wirklichkeit, die durch Symbol gegeben ist, ist eine zergliederte, geordnete und umgestaltete Wirklichkeit. Jedes Symbol ist nach Losev auch eine Darstellung: Es ist geformt und geordnet, sofern die Konstruktion, deren Gesetz das Symbol enthält, eine geordnete Darstellung des konstruierten Gegenstandes ist. Der Träger dieser Ordnung ist das Gesetz, weil es selbst als ein System von Operationen auf bestimmten Objekten aufgefasst werden kann.

Sofern die Wirklichkeit, die durch Symbole konstruierbar ist, die Wirklichkeit der Zeichen sein kann, ist die Losevsche Auffassung des Symbols auf die Symbole anwendbar, die den Bestand des logischen Symbolismus nach Wittgenstein bilden. Die Möglichkeit dieser Anwendung ist dadurch

${ }^{22}$ E. Cassirer. Philosophie der symbolischen Formen I. Darmstadt, Wissenschaftliche Buchgesellschaft, 1972, 5. Aufl., 43

${ }^{23}$ A.F. Losev. Das Problem des Symbols und die realistische Kunst (russ.). Moskau, Iskusstvo, 1976 
gegeben, dass Wittgenstein erstens jedem Symbol eine zeigende Funktion zuspricht und zweitens das Realisieren dieser Funktion als Operieren auf Symbolen auffasst ${ }^{24}$. Von jedem Symbol des logischen Symbolismus kann man behaupten, dass es ein gewisses Gesetz seiner eigenen Transformation oder der Transformation anderer Symbole enthält und somit bestimmt, wie man mit seiner Hilfe neue Symbole gewinnt. Als ein System von Operationen betrachtet, setzt ein solches Transformationsgesetz erstens eine bestimmte Vielfachheit der zur Durchführung einer jeden Operation notwendigen Zeichen (der Basen der Operation) und zweitens eine bestimmte Vielfachheit und einen bestimmten Charakter der Operation selbst voraus. Dass ein Symbol die Möglichkeit der Anwendung von Operationen zeigt, indem es seinen Zusammenhang mit anderen Symbolen zeigt, kann als seine symbolisierende Funktion definiert werden. Die Anwendung der Auffassung Losevs auf die Rekonstruktion der Theorie des logischen Symbolismus ermöglicht zunächst die Erklärung einiger Thesen Wittgensteins. So behauptet Wittgenstein 1914, dass Symbole wie logische Sätze alle auf dieselbe Weise zeigen, nämlich dadurch, dass sie Tautologien $\operatorname{sind}^{25}$. Was sie zeigen, unterscheidet sich aber vom Symbol zum Symbol. Dieses Verschiedene lässt sich nun als Gesetz definieren, das eine Regel des logischen Schließens vorgibt und einen nach der Regel vollzogenen Schluss von einem jeden Schluss nach einer anderen Regel unterscheidet.

\section{$\S 1 \mathrm{c}$. Der logische Symbolismus nach Wittgenstein}

Dass die Losevsche Auffassung des Symbols auf die Theorie Wittgensteins anwendbar ist, möchte ich durch die Analyse des Gebrauchs des Symbolbegriffs im Tractatus begründen. Das Symbol im Sinne Wittgensteins wird in der Regel als Zeichen in seiner projektiven Beziehung zur Wirklichkeit oder als Zeichen zusammen mit seinem Sinn oder seiner Bedeutung verstanden $^{26}$. Diese Auffassung beruht auf einigen Thesen des Tractatus und wird auch durch spätere Manuskripte Wittgensteins bestätigt ${ }^{27}$, bewirkt

\footnotetext{
${ }^{24}$ TLP, 4.122, 5.2-5.21

${ }^{25}$ Notes Dictated to Moore, 114

${ }^{26}$ S. z.B. M. Black. A Companion to Wittgenstein's 'Tractatus' (im weiteren:

Black.Companion). Cambridge, University Press, 1971, 130.

${ }^{27}$ Vgl. VI.Bemerkungen, 206, 248.
} 
aber, dass man das Satzzeichen im Gegensatz zum Satz mit einem fast selbständigen von dem Sein des Satzes unabhängigen Sein belegt. Diese Betrachtungsweise wird z.B. von Black (1971) vertreten, der zwischen Bild als Fakt (picture-vehicle) und Bild als Bild unterscheidet. Laut Auffassung Blacks wird ein Bild vom Fakt zum Bild, das abbildet, wenn seine Elemente auf bestimmte Weise zu Gegenständen zugeordnet werden ${ }^{28}$. In Hinblick darauf, dass Zuordnung durch Namen geschieht und der Satz unabhängig von der Situation seines Gebrauchs betrachtet wird, bedeutet diese Auffassung des Satzzeichens, dass es ein unvollständiges Bild oder eine Satzfunktion im Sinne Russells ${ }^{29}$ ist: Das so verstandene Satzzeichen muss nicht nur Abzeichen für alle denkbaren Wahrheitsmöglichkeiten des Satzes, sondern auch freie Variablen enthalten, was jeden in dem logischen Symbolismus konstruierbaren Satz zum Wert einer Satzfunktion macht.

Die Identifizierung des Symbols mit einem sinnvollen oder bedeutungsvollen Zeichen ist nicht nur infolge solcher Implikationen, sondern bereits deswegen nicht möglich, weil auch sinnlose Zeichen wie Tautologien und Kontradiktionen laut dem Tractatus nicht nur zum Symbolismus gehören ${ }^{30}$, sondern auch selbst Symbole sind ${ }^{31}$. Symbole wie sinnvolle Sätze kennzeichnen Form und Inhalt. Die von einem Symbol gekennzeichnete Form unterscheidet sich von seinem Inhalt dadurch, dass die Form im Symbol selbst enthalten ist. So ist im Satz die Form seines Sinnes enthalten ${ }^{32}$. Der Inhalt ist dagegen im Satz nicht enthalten. Da im sinnvollen Satz nicht das Projizierte enthalten ist, kann man vor allem das Projizierte ${ }^{33}$ mit dem Inhalt des Satzes identifizieren. Das Projizierte ist für den sinnvollen Satz der Satz-Sinn, den Wittgenstein in Sätzen 2 und 3 des Tractatus als die vom Satz abgebildete Sachlage charakterisiert ${ }^{34}$. Ist der Sinn des Satzes eine mögliche Sachlage, besteht die projektive Beziehung des Satzes zur Wirk-

\footnotetext{
${ }^{28}$ Black.Companion, 81, 100

${ }^{29}$ Russell und Whitehead gebrauchen Begriffe ,proposition“" und ,propositional function“ auch auf die Weise, welche die Übersetzung dieser Begriffe als „Satz“ und „Satzfunktion“ erlaubt. Vgl. Erstes Kapitel.

${ }^{30}$ TLP, 4.4611

${ }^{31}$ Ebd., 6.113

${ }^{32}$ Ebd., 3.31, 3.13

${ }^{33}$ Ebd., 3.12-3.13

${ }^{34}$ Ebd., 2.202, 2.221, 3.11, 3.13
} 
lichkeit darin, dass der Satz die Wirklichkeit ,auf ja oder nein fixiert ${ }^{\star 35}$, d.h. das Bestehen eines Sachverhaltes bejaht oder verneint und somit eine der möglichen Sachlagen auswählt. Sofern eine Sachlage mit einer Tatsache, d.h. mit dem Bestehen oder Nichtbestehen von Sachverhalten gleichgesetzt werden kann ${ }^{36}$, und die Rede von möglichen Sachlagen somit nur ein Mittel ist, von der Möglichkeit des Bestehens oder Nichtbestehens von Sachverhalten zu reden, ohne diese Möglichkeiten als Möglichkeiten ausdrücklich zu erwähnen, kann man den Inhalt des Satzes sogar mit der von dem Satz (richtig oder falsch) abgebildeten Tatsache gleichsetzen.

Wenn das Symbol alles ist, was für den Sinn des Satzes wesentlich ist und was alle Symbole, die denselben Zweck erfüllen, gemeinsam haben ${ }^{37}$, kann man versuchen, die vom Symbol gekennzeichneten Form und Inhalt für verschiedene Arten von Symbolen zu definieren.

Das Wesentliche an solchen Symbolen wie sinnvolle Sätze ist das, was allen Sätzen mit demselben Sinn gemeinsam ist. Diese Gemeinsamkeit erlaubt es, einen gegebenen Satz durch einen anderen mit demselben Sinn zu ersetzen $^{38}$. So kann man den Satz „, p“ durch den Satz „, p“ ersetzen. Was diesen beiden Sätzen gemeinsam ist, ist der von ihnen gekennzeichnete Inhalt, der mit der von jedem von ihnen abgebildeten Tatsache des Nichtbestehens eines Sachverhaltes $p$ identifizierbar ist, und die von ihnen gekennzeichnete Form - die Form eines sinnvollen verneinenden Satzes. Das Gemeinsame der beiden Sätze erkennt man, wenn man erkennt, dass jedem von ihnen ein und derselbe Satz „p“ widerspricht. Nach Wittgenstein handelt es sich bei den beiden Sätzen um ein und denselben Satz.

Will man Form und Inhalt auch für Symbole definieren, die keine sinnvollen Sätze sind, ist ihr Inhalt nicht als Projiziertes definierbar, weil solche Symbole keine logischen Bilder sind. Dass solchen Symbolen ein bestimmter Inhalt zugeordnet werden kann, darf nicht mit der Annahme verwechselt werden, dass sie eine semantische Funktion in bezug auf das ihnen Zugeordnete erfüllen und es z.B. bezeichnen oder ausdrücken. Durch diese Annahme wird der Inhalt des Symbols vergegenständlicht und so mit dem Sinn eines Satzes oder mit der Bedeutung eines Namens vergleichbar

\footnotetext{
${ }^{35}$ Ebd., 4.023

${ }^{36}$ Ebd., 2.11

${ }^{37}$ Ebd., 3.31, 3.341

${ }^{38}$ Ebd., 3.341, 3.344
} 
sein. Legt man der Feststellung des Inhalts solcher Symbole ihre Gemeinsamkeiten zugrunde, kann man den Inhalt des Symbols als das charakterisieren, was ein gegebenes Symbol von einem anderen von dem gegebenen verschiedenen Symbol, das dieselbe symbolische Funktion erfüllt, unterscheidet. Sofern symbolisiert formale Eigenschaften und Beziehungen werden, ist der Inhalt des Symbols die Voraussetzung der Vielfältigkeit innerhalb einer jeden durch die Form des Symbols bestimmten Art formaler Beziehungen. Der Inhalt des Symbols ist von seiner Form untrennbar: Man kann formale Beziehungen der logischen Folgerung zwischen zwei Sätzen von den konstruktiven Beziehungen zwischen denselben Sätzen und ihren Wahrheitsargumenten unterscheiden, aber es gibt keine Beziehung der logischen Folgerung, welche nicht eine bestimmte Schlussregel realisiert, und keine Abhängigkeit der Wahrheitsfunktion von ihren Wahrheitsargumenten, welcher nicht die Anwendung einer bestimmten Wahrheitsoperation zugrunde liegt. Wenn man davon ausgeht, dass die symbolische Funktion eines Zeichens im Symbolismus durch seine Verwendung geregelt wird, kann man die Form als eine bestimmte Verwendungsweise von dem Inhalt als bestimmten Verwendungsbedingungen des Symbols unterscheiden.

Man kann als Wesentliches eines jeden Symbols, auch des Symbols, das kein sinnvoller Satz ist, das Gemeinsame, das alle Symbole, die denselben Zweck erfüllen, haben, betrachten und dieses Gemeinsame als Grund der gegenseitigen Ersetzbarkeit der Symbole ansehen.

Das von Wittgenstein als ein Symbol einer Formenreihe charakterisierte ${ }^{39}$ Zeichen für die Regel der Konstruktion der zusammengesetzten Sätze (die im Satz 6 des Tractatus definierte allgemeine Form des Satzes) dient der Konstruktion von Symbolen, d.h. der Angabe einer logischen Notation. Das, was die Ersetzung eines auf diese Weise verwendbaren Symbols durch ein anderes Symbol erlaubt, ist das Gemeinsame des Symbols der Formenreihe der Sätze und der Symbole, die es ersetzen können. Das Symbol, das eine Regel ausdrückt, nach welcher sämtliche Symbole einer logischen Notation aufgebaut werden, kann mithin nur durch den Ausdruck der Konstruktionsregel eines logischen Symbolismus ersetzt werden. Eine solche Ersetzung unterliegt gewissen Einschränkungen: Wird z.B. eine lo-

${ }^{39}$ Ebd., 3.313, 3.314, 5.2522, II.Bemerkungen, 135 
gische Notation durch die Anwendung des Konjunktionszeichens als der einzigen logischen Konstante aufgebaut, kann die Konstruktionsregel für eine solche Notation nicht die Regel ersetzen, welche die Konstruktion von Wahrheitsfunktionen durch die Anwendung des Disjunktions- und des Negationszeichens regelt. Die Ersetzung ist nur dann möglich, wenn beide Symbole Ausdrücke einer Konstruktionsregel sind und die Mengen der Wahrheitsfunktionen, die man aus einer gegebenen Menge von Elementarsätzen durch die Anwendung der beiden Regeln gewinnt, zusammenfallen. Dieses Zusammenfallen wird in den Regeln festgehalten, nach welchen Wahrheitsfunktionen, die in einer Notation konstruierbar sind, in die Wahrheitsfunktionen überführt werden, die zu einer anderen Notation gehören ${ }^{40}$. Sofern der Ausdruck einer Konstruktionsregel den Bezug der Wahrheitsoperationen auf ihre Basen zeigt und Symbole für die Basen angibt, kann man die Form einer Konstruktionsregel, d.h. den Umstand, dass ihr Symbol als Konstruktionsregel gebraucht wird, als die von dem Symbol gekennzeichnete Form definieren ${ }^{41}$. Als den von dem Symbol gekennzeichneten Inhalt kann man die beiden Satzmengen, die der Elementarsätze und die der konstruierbaren Wahrheitsfunktionen, auffassen. Gewisse Satzmengen können auch insofern als Inhalt des Symbols einer Konstruktionsregel betrachtet werden, als sie im Symbol selbst nicht enthalten sind. Tautologien definieren bejahende Operationen, d.h. Operationen des logischen Schließens. Was die Operationen des logischen Schließens kennzeichnen, kann man, der Behauptung Wittgensteins folgend ${ }^{42}$, als Unterschied von Formen definieren, der zwischen dem Satz, der als Prämisse einer durch eine Tautologie definierten Schlussregel auftritt, und dem Satz, der als Schlusssatz nach der Regel gewonnen wird, besteht. Die Frage, wann man eine Tautologie durch eine andere ersetzen kann, stellt sich vor allem für das logische Schließen von einem sinnvollen Satz auf einen anderen sinnvollen Satz. Will man eine Schlussregel durch eine andere Schlussregel ersetzen, müssen die Schlüsse bei der Anwendung der beiden

\footnotetext{
${ }^{40} \mathrm{Vgl}$. TLP, 3.3441, 5.51.

${ }^{41}$ Diese Definition ist insofern möglich, als eine Regel des Aufbaus eines logischen Symbolismus die konstruktiven Operationen des Symbolismus definiert und jede Operation laut Wittgenstein keine Form kennzeichnet, sondern den Unterschied der Formen der Basen und des Resultats der Operation. S. TLP, 5.241.

${ }^{42}$ TLP, 5.241
} 
Regeln auf dieselben Prämissen zusammenfallen. Als eine Schlussregel kann man auch eine Menge von Tautologien betrachten. Die Elemente einer solchen Menge kann man zu einer Konjunktion zusammenfassen, die wegen des tautologischen Charakters der Konjunkte wiederum eine Tautologie wäre. Wenn Wittgenstein behauptet, dass der Satz jeden Satz bejaht, der aus ihm folgt, charakterisiert er das logische Folgen als Beziehung zwischen einem Satz und einer Satzmenge. Davon ausgehend, kann man jeder Schlussregel eine Menge von Satz-Paaren zuordnen. Der Regel modus po-

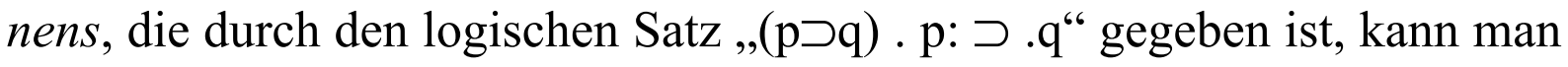

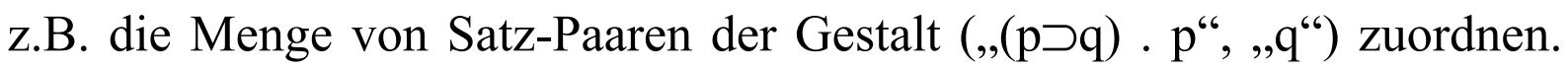
Will man diese Tautologie, d.h. diese Schlussregel, durch eine andere ersetzen, muss die ersetzende Schlussregel dieselbe Menge von Satz-Paaren definieren. Für den modus ponens ist dieselbe Menge z.B. durch die Menge

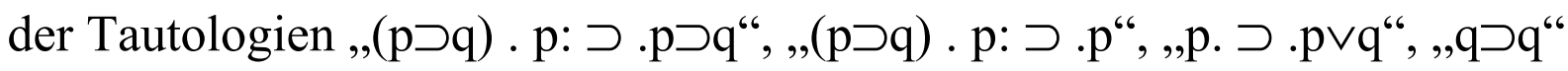

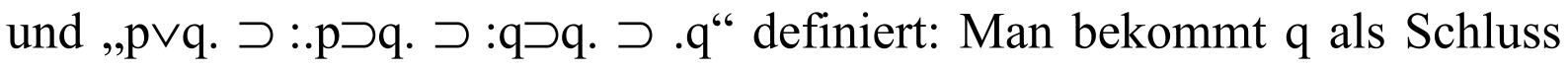
aus der Prämisse ,(pつq) . p“ sowohl durch die Anwendung des modus ponens, als auch dadurch, dass man die beiden Konjunkte ,p“ und „p $\supset \mathrm{q}$ “ aus

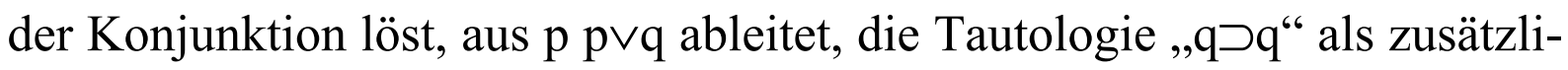
che Prämisse hinzunimmt, und aus der Disjunktion „p pq“ und daraus, dass die beiden Disjunkte q implizieren, $\mathrm{q}$ ableitet. Somit kann man eine Menge von Satz-Paaren als den von einer Tautologie gekennzeichneten Inhalt und die Form einer Schlussregel, d.h. den Umstand, dass die Tautologie eine Tautologie ist, als die von ihr gekennzeichnete Form auffassen.

Die Folgebeziehungen eines sinnvollen Satzes sind seine Beziehungen zu Sätzen, die aus ihm folgen oder ihn implizieren. Diese logischen Beziehungen sind wie die Beziehungen des sinnvollen Satzes zu seinen Wahrheitsargumenten interne Beziehungen des sinnvollen Satzes, die seinen Ort im logischen Raum bestimmen. Sie werden vom Satz selbst sowie von Tautologien gezeigt. Wenn die symbolisierende Funktion eines Satzes seine zeigende Funktion ist, ist ein sinnvoller Satz (ein logisches Bild) in bezug auf seine internen (formalen) Beziehungen zu anderen Sätzen ein Symbol für diese Beziehungen und deshalb für den Ort des Satzes im logischen Raum. Dieser Ort ist durch den Sinn des Satzes insofern bestimmt, als die Wahrheitsargumente des Satzes und seine Wahrheitsbedingungen bestimmen, welche sinnvolle Sätze aus dem Satz folgen können, aus wel- 
chen sinnvollen Sätzen er folgt und welcher Satz ihm widerspricht. Die symbolische Funktion eines sinnvollen Satzes lässt sich zusammen mit seinen anderen semiotischen Funktionen (s. § 1a) mit Hilfe eines Schemas darstellen (Schema 1).

Dass Konstruktionsregeln und Tautologien Symbole in dem Sinn sind, dass sie eine unendliche Bedeutung haben, kann man dadurch begründen, dass man Ausdrücke für Konstruktionsregeln als Regeln der unbeschränkten Konstruktion von Sätzen aus gegebenen Elementarsätzen und Tautologien als Regeln des Gewinnens von Schlüssen verschiedener Form aus den auf diese Weise konstruierbaren Sätzen gebraucht. Einen sinnvollen Satz charakterisiert als Symbol für Unendliches, dass man aus ihm unter der Annahme der unbeschränkten Anzahl der Elementarsätze beliebig viele Weltbeschreibungen (zusammengesetzte Sätze) konstruieren und aus diesen im Fall ihrer Wahrheit Schlüsse ziehen kann. Während Tautologien und Konstruktionsregeln bereits per definitionem Gesetze oder Vorschriften sind, die eine bestimmte Operation vorgeben, beinhaltet ein sinnvoller Satz auch ein bestimmtes Gesetz oder eine Vorschrift. Ein Elementarsatz enthält z.B. die Regel seiner Verallgemeinerung, weil er zeigt, nach welchen Regeln die Namen im Satz und folglich die den Namen entsprechenden Elemente der Struktur der Tatsache zusammenhängen, ein zusammengesetzter Satz enthält die Vorschrift, nach welcher er analysiert wird und zugleich die Regel, nach welcher man seine logischen Folgerungen oder Prämissen suchen kann, ein jeder Satz enthält die Vorschrift, ihn als eine Wahrheitsfunktion zu behandeln, die zur Konstruktion weiterer Sätze gebraucht werden kann.

Aufgrund dieser Rekonstruktion der Theorie Wittgensteins kann man auch bestimmen, welchen Bestand der anwendbare logische Symbolismus hat. Symbole (Elemente des Symbolismus) umfassen Namen, sinnvolle Sätze, die aus Namen bestehen, Tautologien sowie Definitionen und Regeln der Konstruktion des Symbolismus. Sofern Definitionen und Tautologien für ihre Formulierung der Variablen bedürfen, enthält Symbolismus auch Variablen sowie die die Formen von Elementarsätzen angebenden Satzformen. Variablen und Satzformen sind insofern Symbole, als sie interpretiert werden, d.h. als Gegenstands-, Funktionsvariablen, Wahrheitsfunktionen vertretende Aussagevariablen und Satzformen und folglich als Träger von Zeichenzusammenhängen verstanden werden. 


\section{$\S 2$. Die Problematik der Bildtheorie}

Wittgensteins Charakterisierung der Symbole durch die Unterscheidung ihrer zeigenden und sagenden Funktionen weist Parallelen zu einer anderen Charakterisierung der logischen Symbole auf. Bereits 1847 unterscheidet Boole zwischen darstellenden und Operations- Funktionen eines Symbols. Symbole für Individuen stellen nur dar, während andere Symbole sowohl darstellen als auch operieren. So stellen Symbole für Klassen Klassen dar und operieren auf Symbolen für Individuen, indem sie einige von diesen als Elemente einer Klasse auswählen ${ }^{43}$. Die Elemente des Symbolismus nach Wittgenstein kann man auch zunächst in sinnvolle oder sagende (sinnvolle Sätze) und vertretende (Namen) einerseits und zeigende Symbole wie Tautologien andererseits unterteilen. Diese Unterteilung ist aber nicht dichotomisch, sofern auch sinnvolle und vertretende Symbole Symbole in dem Sinn sind, dass sie etwas zeigen. Auch einfache Symbole Namen vertreten nicht nur. Sofern sie für den Sinn des Satzes wesentlich sind, sind sie Ausdrücke oder Symbole, die außer Inhalt auch Form kennzeichnen und formale Eigenschaften haben, die durch ihren logischsyntaktischen Gebrauch erkannt werden. Vor allem zeigen die Namen, dass sie einen Gegenstand bezeichnen ${ }^{44}$. Diese symbolische Funktion von Namen bestimmt größtenteils die Problematik der Bildtheorie.

Das erste Problem verbirgt sich in der Charakterisierung der Form der Abbildung. Dass die Form der Abbildung als gemeinsame Form des Bildes und des Abgebildeten und zugleich als die Möglichkeit der Struktur des Bildes, die durch den Bezug auf die Elemente des Bildes charakterisiert ist, definiert wird, bedeutet, dass das Gemeinsame des Bildes und der Wirklichkeit einem nur als Form der Konstruktion des Bildes bekannt ist oder dass die abgebildete Wirklichkeit von der Konstruktion des Bildes nicht unabhängig ist, sondern mit ihm mitkonstruiert wird. Im Tractatus wird die Anerkennung dieser Abhängigkeit durch die These bestätigt, dass eine physikalische Weltbeschreibung verfeinert werden kann und eine verfei-

${ }^{43}$ G. Boole. The Mathematical Analysis of Logic (im weiteren: Boole.Analysis). In: G. Boole. Collected Logical Works I. La Salle, Illinois, Open Court Publishing Company, 1952, 49-124, 60-63

${ }^{44}$ TLP, 4.126 
nerbare Beschreibung eine Beschreibung des Netzes ist, das auf die Welt angelegt wird, um die Beschreibung zu ermöglichen ${ }^{45}$. Die Frage, die sich hier stellt, ist folgende: Wenn die Elemente des Bildes (insbesondere die Namen in einem Satz), die sich zu einander in einer bestimmten Weise verhalten, Gegenstände vertreten, die Bestimmung der Gegenstände jedoch durch die Konstruktion des Bildes bedingt ist, welchen Charakter hat diese Vertretung und wie kommt sie zustande? Wenn das Zustandekommen dieser Vertretung derjenige bestimmt, der das Bild in jedem Einzelfall konstruiert, was bürgt dafür, dass die Elemente des Bildes und folglich die Gegenstände für jeden, der den Satz formuliert oder ihn versteht, dieselben sind? Anders ausgedrückt, was macht den Symbolismus, der zum Zweck der Konstruktion eines Bildes, eines Modells, einer Weltbeschreibung aufgebaut wird, zu einem im eigentlichen Sinn logischen Symbolismus, der von mehr als nur einem benutzt werden kann, um die Welt zu beschreiben und aus ihrer Beschreibung Schlüsse zu ziehen? Ist die Bestimmung der vertretenden Funktion von Namen von einem einzelnen den Satz Formulierenden unabhängig, wie sind dann einem die Namen und die die Vertretung regelnde Zuordnungsvorschrift gegeben? Diese Fragestellung ist im Tractatus dadurch entschärft oder verborgen, dass Wittgenstein, wie es seine späteren Manuskripte zeigen, zwischen einer phänomenologischen und einer physikalischen Beschreibungsweise und Sprache unterscheidet und die phänomenologische Beschreibungsweise der Welt für primär hält, so dass die Besonderheiten der physikalischen Beschreibungsweise nicht jede abbildende Beziehung charakterisieren müssen. Mit der Anerkennung dessen, dass jede Beschreibung der Sprache die Beschreibung eines physikalischen Phänomens ist, verzichtet Wittgenstein später darauf, den primären Charakter einer Beschreibungsweise oder Sprache anzuerkennen, und wendet sich der gewöhnlichen Sprache zu, die er auch für phänomenologisch hält ${ }^{46}$.

Das zweite Problem betrifft den Charakter der Zuordnungen zwischen Namen und Gegenständen. Falls der logische Symbolismus Namen enthält, geht dem Aufbau der logischen Syntax die Zuordnung der Namen zu Ge-

\footnotetext{
${ }^{45}$ Ebd., 6.341, 6.35

${ }^{46}$ II.Bemerkungen, 190-191, L. Wittgenstein. „Philosophische Betrachtungen“ (im weiteren: Betrachtungen). In: L. Wittgenstein. Wiener Ausgabe 2. Wien, New York, Springer, 1994, 3-203, 4
} 
genständen voraus, sofern die Angabe der allgemeinen Form des Satzes in Abhängigkeit von der Wahl der Namen gesetzt wird ${ }^{47}$. Diese Zuordnung geschieht durch Erläuterungen der Bedeutungen von Namen. Zugleich werden Namen zur Konstruktion der logischen Bilder (Sätze) gebraucht, und diesen Gebrauch kann man selbst als eine Zuordnung deuten, für welche Namen als Elemente der Zuordnungsvorschrift auftreten ${ }^{48}$. Die Formulierung der Elementarsätze besteht also in einer Zuordnung, die selbst auf einer Zuordnung basiert. Die Konstruktion eines logischen Bildes ist somit keine direkte ${ }^{49}$ Darstellung der Wirklichkeit oder direktes Abbild einer Tatsache, sondern beruht bereits auf einer Konvention und ist durch diese vermittelt. Wenn eine Tatsache logisch abbilden zu sagen, wie ein Gegenstand ist, bedeutet, sind die Konventionen über die Bedeutungen von Namen, die der Abbildung (der Formulierung des Satzes) zugrunde liegen, vor-logisch?

Jede Konstruktion eines logischen Bildes kann als eine durch den logischen Symbolismus geregelte Konstruktion aufgefasst werden. Sofern die Elementarsätze einem vor der Formulierung des logischen Symbolismus gegeben sind und die Logik nur darüber entscheidet, welche Elementarsätze sie in ihren Symbolismus aufnimmt, um sie zu verwenden, entscheidet die Logik auch über die analysierte Form dieser Sätze ${ }^{50}$, was bedeutet, dass sie den Ausdruck dieser Form mittels einer Satzvariablen, d.h. einer Kombination von Variablen wie „fx“ durch eine syntaktische Regel vorgibt. Dann besteht jede Konstruktion eines Elementarsatzes im Rahmen des Symbolismus darin, dass die Variablen in dem Ausdruck einer solchen Form durch Namen ersetzt werden. Dem Resultat einer solchen Konstruktion kann der Charakter eines logischen Bildes dadurch verliehen werden, dass man die Übereinstimmungsbedingungen des Resultats der Konstruktion mit der Wirklichkeit definiert. Eine solche Definition wird z.B. von Ricketts $(1996)^{51}$ vorausgesetzt, der keine besonderen Regeln in Form der der Formulierung von Sätzen vorausgehenden Zuordnungen von Bedeu-

${ }^{47}$ TLP, $3.33,4.5$

${ }^{48}$ Auf diese Möglichkeit weist insbesondere Stenius hin. Stenius.Tractatus, 130-132

${ }^{49}$ Die Meinung über den direkten Charakter der Darstellung der Wirklichkeit durch das logische Bild vertritt z.B. Toulmin. S. Toulmin. „Ludwig Wittgenstein“ (im weiteren: Toulmin.Wittgenstein). In: Encounter XXXII, 1969, 58-71, 67.

${ }^{50} \mathrm{Vgl}$. TLP, 5.5562, 5.557.

${ }^{51}$ S. Ricketts.Pictures. 
tungen zu Namen anerkennt. Laut Ricketts gibt es nur Projektionsregeln, welche die Bedingungen der Übereinstimmung der Sätze mit der Wirklichkeit in einen Zusammenhang mit der Angabe der Bedeutungen von Namen bringen. Deswegen sind Zuordnungen von Namen und Gegenständen nicht-extensionale Zuordnungen, die durch solche Regeln geregelt werden. Diese Auffassung des Charakters der logischen Abbildung kann man durch die These, dass die Bedeutungen von Namen durch Sätze erklärt werden, und somit durch die Möglichkeit, Namen als durch Sätze vermittelt anzusehen, begründen. So kann man laut Apel (1966) Gegenstände nur im Zusammenhang von Sachverhalten denken, was bedeutet, dass die der Sprache und der Welt gemeinsame logische Form die Regel der syntaktischen Kombination von Sprachzeichen und zugleich eine Vorschrift, welche die kategoriale Form der zu beschreibenden Tatsache vorschreibt, ist ${ }^{52}$. In Hinblick auf die Konstruktion eines logischen Symbolismus stellt sich aber die Frage, welchen Charakter die Projektionsregeln haben. Als Regeln sind die Projektionsregeln entweder Konventionen des Symbolismus selbst und folglich nach Wittgenstein syntaktische Konventionen, die nichts mit der Bedeutung von Zeichen zu tun haben, oder wiederum vor-logische Konventionen. Im ersten Fall ist die Aufgabe der Anwendbarkeit des Symbolismus, streng genommen, nicht gelöst. Der zweite Fall tritt insofern ein, als die Projektionsregeln die Bedingungen der Übereinstimmung der Sätze mit der Wirklichkeit formulieren, indem sie die Sätze selbst für die Formulierung der Übereinstimmungsbedingungen gebrauchen, während Aussagen über Zusammenhänge der den Sätzen entnommenen Bestandteile diese Bestandteile als Namen in den Symbolismus einführen. Ricketts' Projektionsregeln sind mit den Sätzen der Gestalt „'p' sagt p“ vergleichbar: Sie setzen an die Stelle des Namens des Satzes in diesem Ausdruck die Beschreibung einer Beziehung zwischen Bestandteilen des Satzes „p“ und somit einen metasprachlichen Ausdruck, der die Bestandteile des Satzes als Namen in den Symbolismus einführt, und an die Stelle des Satzes „p“ „dass p“, womit der Satz „p“ selbst für die Formulierung der Übereinstimmungsbedingungen gebraucht wird und das Verstehen von $\mathrm{p}$ stillschweigend angenommen wird. Das bedeutet, dass dem Symbolismus die Konventionen des gewöhnlichen Sprachgebrauchs zu-

${ }^{52}$ Apel.Wittgenstein, 52-53 
grunde gelegt werden, so dass die logischen Konstruktionen sich dann auf eine vor-logische sprachliche Begriffsbildung stützen. Sofern Wittgenstein den Satzbegriff dadurch einführt, dass er den Satz dem Namen entgegensetzt, und den Namen durch seine Rolle in einem Satz charakterisiert, setzen die beiden Auffassungen - dass das Verstehen des Sinnes des Satzes die Kenntnis der Bedeutungen von Namen verlangt einerseits und dass die Namen einem nur im Zusammenhang eines Satzes gegeben sind andererseits - einander voraus, was man auch dadurch erkennt, dass die Forderung, Projektionsregeln an die Stelle von Zuordnungsregeln zu setzen, keinen entscheidenden Fortschritt in Hinblick auf die Problematik der Zuordnung bedeutet.

Sofern der logische Symbolismus Namen beschreibt ${ }^{53}$, regelt er die Konstruktion der Elementarsätze. Diese Konstruktion besteht in der Transformation einer Klasse von Namen ${ }^{54}$, d.h. einer Klasse von Symbolen, in ein neues Symbol - einen sinnvollen Satz. Eine solche Transformation, welche die Konstruktion eines Satzes, d.h. eines logischen Bildes, ist, muss eine logische Operation sein, sofern sie durch die logische Syntax geregelt ist. Der logische Charakter der Operation der Konstruktion eines Elementarsatzes ist insofern nicht ausgeschlossen, als die Konstruktion der zusammengesetzten Sätze eine logische, nämlich eine Wahrheitsoperation ist. Darüber hinaus erlaubt der Symbolismus die Verallgemeinerung der Elementarsätze. Die Verallgemeinerung kann als eine Operation des logischen Schließens betrachtet werden. Ein Beispiel für einen Verallgemeinerungsschluss ist der Schluss von der Wahrheit eines Elementarsatzes der Gestalt

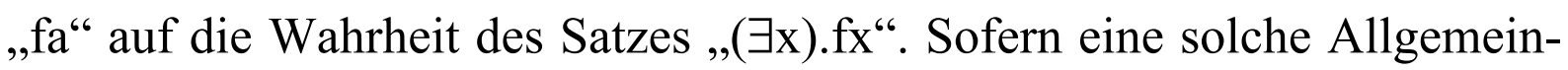
heitsbezeichnung eine Satzvariable „fx“ enthält, muss der Symbolismus entweder derartige Satzvariablen einschließlich der Satzvariablen wie „Nfx“, ohne die kein Allsatz konstruierbar ist, als primitive Symbole enthalten, oder Ersetzungsregeln beinhalten. Den logischen Status der Ersetzungsoperationen definiert Wittgenstein nicht, obwohl er das Ersetzen eines Symbols durch das gleichbedeutende erlaubt und Definitionen in Form von Gleichungen als Zeichenregeln für solche Ersetzungen betrachtet. Dass Wittgenstein zulässt, dass man die Welt auch ohne Zuordnung von

\footnotetext{
${ }^{53}$ TLP, 3.33

${ }^{54}$ Vgl. ebd., 3.142 .
} 
Namen zu Gegenständen beschreiben kann ${ }^{55}$, bedeutet, dass die zeigende Funktion auch von variablen Namen erfüllt wird, die sich als Satzvariablen auffassen lassen und somit Klassen von Sätzen, in welchen sie vorkommen können, und dementsprechend auch bestimmte Operationen, die in erster Linie die Operation der Substitution, die nach Curry im Einsetzen von Konstanten für Variablen in Ausdrücken für propositionale Funktionen besteht ${ }^{56}$ und die von Wittgenstein als eine logische Operation nicht definiert ist, einschließen, definieren. Das Problem des Charakters der Konventionen über Zuordnungen von Namen zu Gegenständen ist somit mit einem weiteren Problem verbunden - dem Problem des Charakters der Operationen, die durch Namen und variable Namen definiert sind.

Dass Wittgenstein sich dieser Problematik bewusst ist, zeigen seine Manuskripte aus den Jahren 1929-1932, in welchen sich der Übergang zu seiner Spätphilosophie andeutet. Zunächst bezweifelt Wittgenstein den Grundcharakter der Elementarsätze. Als Beispiele für Elementarsätze betrachtet er Sätze „hier ist rot“ und „hier ist grün“ und versucht, das Problem ihrer gegenseitigen logischen Abhängigkeit zu lösen. Insbesondere versucht er „hier“ durch die Einführung eines Koordinatensystems zu ersetzen und „rot“ als Kombination von vier Grundfarben darzustellen. Die Möglichkeit solcher Konstruktionen innerhalb der Elementarsätze ist für Wittgenstein die Möglichkeit einer logischen Konstruktion (und folglich einer Operation), die nicht mit Wahrheitsfunktionen arbeitet ${ }^{57}$. Seine Überlegungen kann man folgendermaßen zusammenfassen:

1. Wenn man den Sachverhalt mit einer bestimmten Multiplizität der Gegenstände als Sachverhalt mit einer anderen Multiplizität oder sogar als zwei Sachverhalte darstellen kann, wodurch gewinnt die Analyse einer Wahrheitsfunktion ihre Bestimmtheit? Wenn die Analyse des Satzes mit dem Instrumentarium der logischen Wahrheitsoperationen kein Gebilde ergibt, von welchem man behaupten kann, dass es das eindeutige Resultat dieser Analyse ist, was bestimmt dann den Sinn eines Satzes oder die Bedeutung eines Zeichens? Wittgensteins Antwort auf diese Frage besteht darin, dass die Bedeutung eines Zei-

\footnotetext{
${ }^{55}$ TLP, 5.526

${ }^{56}$ H.B. Curry. "An Analysis of Logical Substitution” (im weiteren:

Curry.Substitution). In: American Journal of Mathematics 51, 1929, 363-384, 368-369

${ }^{57}$ II.Bemerkungen, 56
} 
chens nicht durch den Gegenstand bestimmt ist, welchen das Zeichen vertritt, und der Sinn des Satzes nicht durch die abbildende Beziehung des Satzes zur Tatsache, sondern allein durch den Ort des Zeichens im System anderer Zeichen, durch die Regeln, die für das Zeichen, sei es ein Name oder ein Satz, gelten ${ }^{58}$. Das Zeichen wird von einem zeigenden und/oder vertretenden oder sinnvollen Symbol zu einem Zeichen, das nur durch seine Operations-Funktionen bestimmt ist.

2. Wenn es andere logische Operationen als Operationen auf Wahrheitsfunktionen möglich sind, was sind das für Operationen? Bereits 1916 in den Tagebüchern begründet Wittgenstein die Möglichkeit einer Operation durch die strukturelle Ähnlichkeit ihrer Basen und Resultate. Er erwägt sogar, ob es eine Operation gibt, die dadurch, dass sie einen Elementarsatz in einen anderen überführt, es überhaupt ermöglicht, von Elementarsätzen in ihrer Totalität zu sprechen ${ }^{59}$. Die einzige Möglichkeit, aus einem Elementarsatz einen anderen zu gewinnen, besteht darin, dass man einen in einem Elementarsatz vorkommenden Namen durch einen anderen Namen ersetzt. Wenn man die Anerkennung dieser Möglichkeit im Tractatus nur in dem Begriff eines variablen Namens vermuten kann, wird in den Manuskripten die Ersetzung als eine logische Operation betrachtet, indem die Möglichkeit analysiert wird, nicht nur Definitionen eines logischen Symbolismus, sondern auch Tautologien durch Gleichungen auszudrükken. Tautologien in Form von Gleichungen können auch als Schlussregeln auftreten, die dann zu Ersetzungsregeln werden ${ }^{60}$. Die Analyse der Anwendung der Gleichungen in der Mathematik führt Wittgenstein zu der Idee, dass die Gleichung mit einer Zahl vergleichbar ist, die nichts anderes als eine Reihe von Operationen ist, welche die Zahl erzeugen. Wenn die Bedeutung eines Zeichens wie eine Zahl

\footnotetext{
${ }^{58}$ Vgl. L. Wittgenstein. „Bemerkungen V“ (im weiteren: V.Bemerkungen). In: L. Wittgenstein. Wiener Ausgabe 3. Wien, New York, Springer, 1995, 3-143, 95, VI.Bemerkungen, 201.

59 Tagebücher, 23.11.16

${ }^{60}$ L. Wittgenstein. „I. Philosophische Bemerkungen“ (im weiteren: I.Bemerkungen). In: L. Wittgenstein. Wiener Ausgabe 1. Wien, New York, Springer, 1994, 3-34, 7 , II.Bemerkungen, 67
} 
vollständig durch alle für es geltenden Regeln bestimmt ist ${ }^{61}$ und die Gleichung der Ausdruck einer syntaktischen Regel ist, dann ist die durch die Gleichung gegebene gegenseitige Ersetzbarkeit von Zeichen ein Charakteristikum der Bedeutung der Zeichen ${ }^{62}$, d.h. ein Charakteristikum ihrer Grammatik.

3. Wenn Wittgenstein sich dem Problem des Charakters der Konventionen zuwendet, die insofern als vor-logisch bezeichnet werden können, als sie dem Aufbau des logischen Symbolismus vorhergehen, betrachtet er dieses Problem als Problem der Interpretation oder der Zuordnung, das für ihn das Problem des Anknüpfens des Zeichens an die Wirklichkeit ist ${ }^{63}$. Diese Konventionen oder Zuordnungen haben einen sprachlichen Charakter: Zunächst werden sie in Worten gegeben $^{64}$. Darüber hinaus wird wie bei einer Zuordnung, die im Rahmen eines konstruierten Symbolismus auf Zeichen vollzogen wird und nach Zuordnungsregeln geschieht ${ }^{65}$, auch in der gesprochenen Sprache, wo Zuordnung zur Vorbereitung zum Satz und in diesem Sinn zur Sprachlehre im Gegensatz zur Sprachanwendung gehört ${ }^{66}$, nur ein Zeichen einem anderen Zeichen zugeordnet ${ }^{67}$ : Das Sprachzeichen „rot" wird z.B. einem einzelnen Exemplar von Rot zugeordnet, das auch nur ein Zeichen für Rot ist. Dass man durch die Sprache die Kluft zwischen Sprache und Wirklichkeit nicht überwinden kann, äußert sich nach Wittgenstein darin, dass man durch den Satz, den man hört und versteht, nie den durch den Satz dargestellten Sachverhalt als Datum erhält. Deswegen betrachtet Wittgenstein den Satz selbst als ein solches Datum ${ }^{68}$. Dass die Konstruktion der Sätze bereits im Tractatus als eine Konstruktion aufgefasst wird, die durch Namen-Zuordnungen mit Hilfe von Sätzen (Erläuterungen) vermittelt wird, und dass die Namen-Zuordnungen später als Zuordnungen von Zeichen zu Zeichen verstanden werden, erklärt auch die Gleich-

\footnotetext{
${ }^{61}$ II.Bemerkungen, 96, 99, 174, 178

${ }^{62}$ VI.Bemerkungen, 252, 277

${ }^{63}$ Betrachtungen, 182

${ }^{64}$ VI.Bemerkungen, 313

${ }^{65}$ II.Bemerkungen, 39, 77

${ }^{66}$ I.Bemerkungen, 19, VI.Bemerkungen, 285

${ }^{67}$ VI.Bemerkungen, 175

${ }^{68}$ Ebd., 184
} 
setzung der Begriffe mit Begriffswörtern in den Philosophischen Untersuchungen: Wenn man in der Sprache es nur mit der Sprache und der Arbeit der Zeichen zu tun hat ${ }^{69}$, gibt es keinen Grund, eine besondere begriffliche Wirklichkeit anzunehmen - es gibt keine logischen Gebilde, die hinter den Begriffswörtern stehen, weil die Funktion der Begriffsbildung sowie der Begriffsabgrenzung die Sprache selbst, die in den Philosophischen Untersuchungen vor allem als Sprechen aufgefasst wird, erfüllt. Die sprachliche Zuordnung ist nach Wittgenstein symbolisch, d.h. sie verpflichtet einen zu etwas, insbesondere dazu, dass er die Wirklichkeit bei der Formulierung des Satzes sowie den Satz, den er hört, als artikuliert betrachtet: Das Artikulierte dient einem als Vorlage für die Übersetzung, so dass einerseits gemäß den grammatischen Regeln die Wirklichkeit in den Satz übersetzt wird und andererseits von der Vorlage Satz abgelesen wird, wie die Wirklichkeit zu artikulieren ist ${ }^{70}$.

${ }^{69}$ In V.Bemerkungen, 105, schreibt Wittgenstein „Understanding a symbol means to know/knowing/ how it works".

${ }^{70}$ Ebd., 122-123 
weist Identität auf, bildet ab

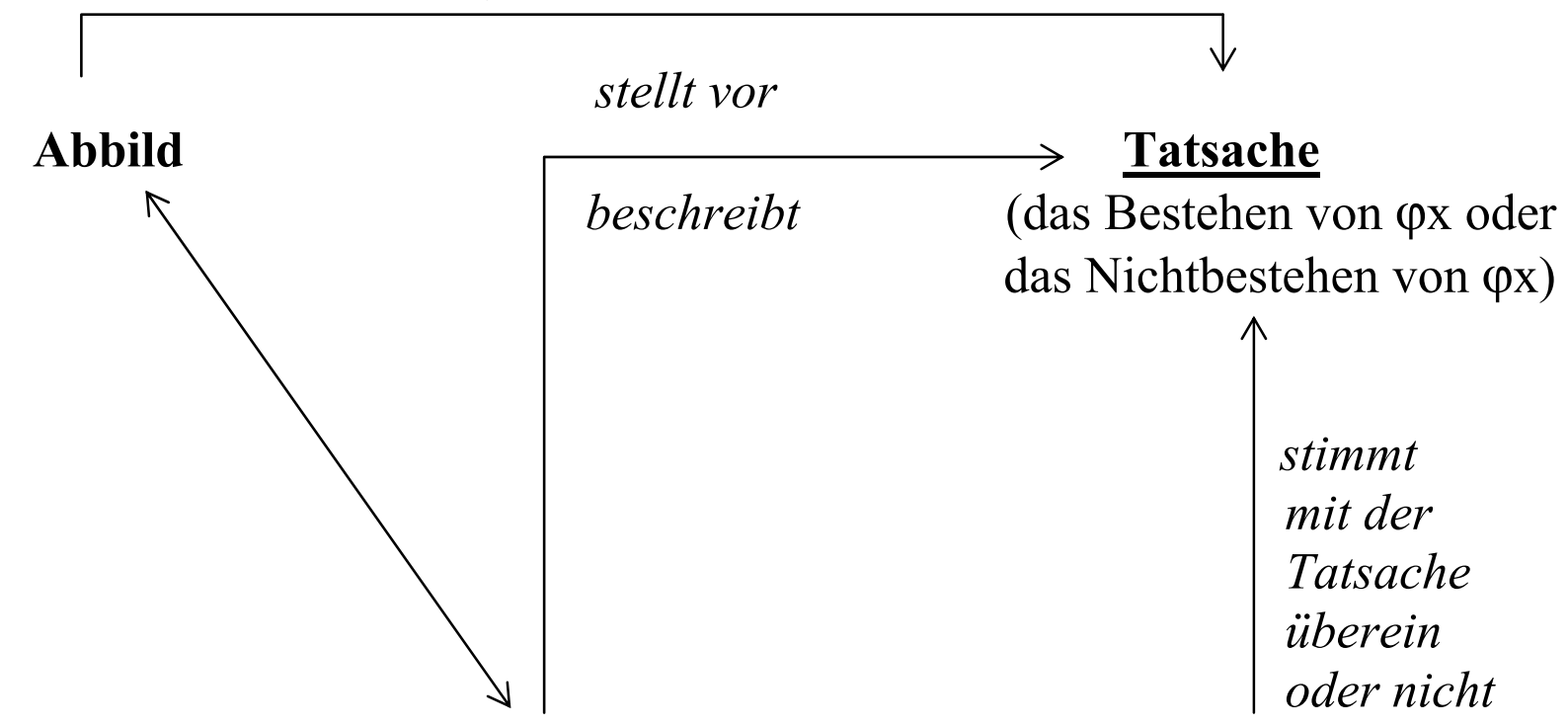

stellt dar

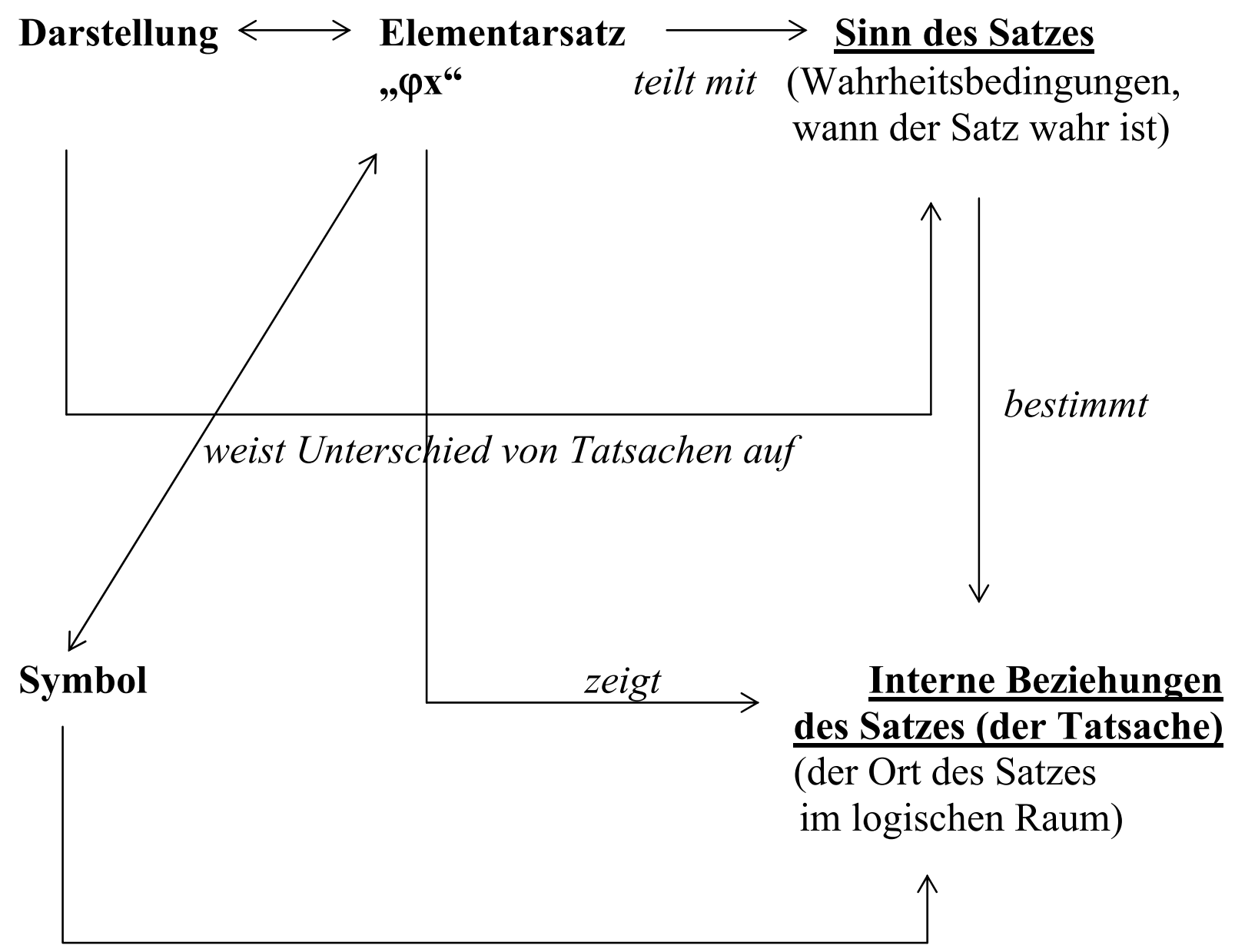

enthält ein Gesetz, nach welchem weitere

Zeichengebilde, z.B. logische Prämissen und

Folgen des Satzes, konstruiert werden können 
